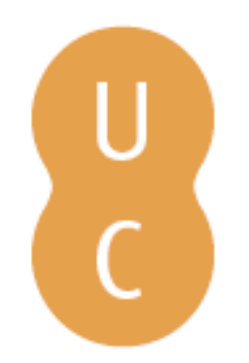

\title{
Rompalina
}

\section{Les intellectuels français et le Portugal de Salazar: mythification et dissonance}

Autor(es): $\quad$ Martins, Otília Pires

Publicado por: Imprensa da Universidade de Coimbra

URL

persistente: URI:http://hdl.handle.net/10316.2/38712

DOI: $\quad$ DOl:http://dx.doi.org/10.14195/978-989-26-1164-8_

Accessed : $\quad$ 26-Apr-2023 13:40:35

A navegação consulta e descarregamento dos títulos inseridos nas Bibliotecas Digitais UC Digitalis, UC Pombalina e UC Impactum, pressupõem a aceitação plena e sem reservas dos Termos e Condições de Uso destas Bibliotecas Digitais, disponíveis em https://digitalis.uc.pt/pt-pt/termos.

Conforme exposto nos referidos Termos e Condições de Uso, o descarregamento de títulos de acesso restrito requer uma licença válida de autorização devendo o utilizador aceder ao(s) documento(s) a partir de um endereço de IP da instituição detentora da supramencionada licença.

Ao utilizador é apenas permitido o descarregamento para uso pessoal, pelo que o emprego do(s) título(s) descarregado(s) para outro fim, designadamente comercial, carece de autorização do respetivo autor ou editor da obra.

Na medida em que todas as obras da UC Digitalis se encontram protegidas pelo Código do Direito de Autor e Direitos Conexos e demais legislação aplicável, toda a cópia, parcial ou total, deste documento, nos casos em que é legalmente admitida, deverá conter ou fazer-se acompanhar por este aviso. 
Otília Pires Martins

Universidade de Aveiro / Centro de Línguas e Culturas (CLC)

\section{LES INTELLECTUELS FRANÇAIS ET LE PORTUGAL DE SALAZAR: MYTHIFICATION ET DISSONANCE ${ }^{1}$}

La force et la faiblesse des dictateurs est d'avoir fait un pacte avec le désespoir des peuples.

(Bernanos, Nous autres Français, Paris, Gallimard, 1939)

\section{Introduction}

$\mathrm{Au} \mathrm{xv}^{\mathrm{e}}$ siècle, les premiers vaisseaux d'Henri le Navigateur quittent Sagres, la pointe la plus occidentale de l'Europe, pour croiser des océans inconnus et menaçants. Le Portugal des Découvertes s'engageait ainsi dans la voie de l'expansion mondiale et lançait les bases du premier Empire européen moderne: il s'ouvrait au monde en même temps qu'il ouvrait, à l'ensemble de l'Europe, les portes de l'Âge Moderne. Puis, cette même nation qui donna "de nouveaux mondes au monde", se referme lentement sur elle-même, jusqu'au xx $x^{\mathrm{e}}$ siècle, où une longue dictature la plonge, pendant près de cinquante ans, dans un isolement triste et sombre.

${ }^{1}$ Je garde en mémoire le souvenir vif et lumineux de ma première rencontre avec Mme Ofélia Paiva Monteiro, à l'Université Catholique de Viseu, en 1985, lors d'une cérémonie d'ouverture de l'année universitaire. Cette rencontre devait porter ses fruits car, peu de temps après, elle acceptait de co-diriger, avec M. Michel Lioure, ma thèse de Doctorat sur Julien Green (que j'ai soutenue en 1993). Son amitié, empreinte d'une immense gentillesse et d'une extrême douceur, est un privilège qui enrichit mon existence depuis de longues années. Comment l'en remercier? 
Après le régicide de 1908, la République portugaise voit le jour, le 5 octobre 1910. La victoire républicaine sur la Monarchie demeure, cependant, instable, fragile: Sidónio Pais, qui forme la República Nova, en décembre 1917, est assassiné l'année suivante; le gouvernement de la Gauche Démocratique s'écroule, début 1925, entraînant la dissolution du parlement et l'instauration de la Dictature Militaire en 1926. En 1928, on confie le ministère des Finances à un professeur de l'Université de Coimbra, lui confiant les pleins pouvoirs en matière budgétaire. En un an, il redresse l'économie, de telle sorte qu'en 1929 il apparaît comme l'homme fort du gouvernement et la seconde moitié des années 30 "voit l'affirmation pleine du pouvoir personnel de Salazar dans une Europe où triomphent les dictateurs et les chefs charismatiques des régimes fascistes et autoritaires. Salazar devient le "chef". ${ }^{2}$ Après l'Italie de Mussolini, l'Allemagne de Hitler et l'Espagne de Franco, c'est au tour du Portugal d'avoir son chef et son régime autoritaire. Salazar a, cependant, idéalisé un fascisme "à la portugaise" - nationaliste, catholique et corporativiste - et un modèle de société - protégée de toute influence étrangère, peu industrialisée, puritaine, paroissiale et disciplinée. L'Estado Novo se donne pour devise le respect de trois notions fondamentales - "Famille, Travail, Patrie" - et comme tout régime autoritaire, il aura une police de sécurité de l'Etat - PIDE - qui protège le régime, à partir de 1933, interrogeant, torturant et emprisonnant les opposants. Elle travaille côte à côte avec les services de censure et dispose du droit de violation du courrier et d'écoutes téléphoniques. Sa répression s'exerce sur tous les fronts - livres, journaux, pamphlets, brochures - qu'il s'agisse de publications nationales ou étrangères -, émissions de radio et, après 1950, de télévision. La liberté d'expression prend subrepticement la forme d'un lointain e inaccessible mirage.

\section{António Ferro et la "politique de l'esprit ${ }^{3}$}

Pour contrebalancer cette censure, il fallait faire appel à un service de propagande politique qui, en même temps qu'il soutenait le régime totalitaire devait

2 Fernando Rosas, "António de Oliveira Salazar", Dicionário de História do Estado Novo, Lisbonne, Círculo de Leitores, 1996, p. 868. en 1932

3 Cette notion, empruntée à Paul Valéry, est utilisée par António Ferro, pour la première fois, 
transmettre, tant sur le plan national qu'international, une image positive du dictateur. Le Secrétariat de la Propagande Nationale (SPN) est créé en 1933. Un ensemble d'interviews à Salazar changera le destin d'un jeune journaliste qui avait soutenu, dans le Diário de Notícias, la thèse d'une médiatisation de l'Estado Novo, à travers "une politique de l'esprit", expression empruntée à Paul Valéry. Salazar se laisse séduire par l'idée et le nomme à la tête du SPN, plus tard, Secrétariat National de l'Information (SNI). António Ferro, qui fut le compagnon de Fernando Pessoa et de Mário de Sá-Carneiro dans 1'aventure futuriste, devient alors un ministre de la culture avant la lettre: "[...] António Ferro s'employait à donner une dimension culturelle au régime dont il assurait la mythologie, en lui fournissant une vitrine artistique, surtout à l'usage de l'étranger - car la culture était [...] la façade visible "lá fora”“. Il lui faudra, pour cela, trouver le juste équilibre entre ses tendances esthétiques plus tournées vers un concept moderniste de l'art et les exigences d'un régime fascisant et conservateur. Sous sa direction, jusqu'en 1950, cette “cellule” de l'Etat réussira à donner une nouvelle image du Portugal.

Propagandiste acharné, sur le plan national mais surtout à l'étranger, au service de la construction d'une image idéalisée et mythifiée de Salazar et de l'Estado Novo, il crée des Casas de Portugal; il invite des figures de l'Intelligentsia européenne, écrivains et journalistes reconnus, en voyage officiel au Portugal qui, pour la plupart, exalteront, dans leurs écrits, la figure du dictateur et souligneront le "miracle portugais". Il fera traduire les Discours de Salazar et écrira Salazar. Le Portugal et son chef, traduit dans plusieurs langues et préfacé par Paul Valéry ${ }^{5}$. Soulignons, toutefois, que Paul Valéry ne fait, nullement, l'éloge de Salazar, qu'il nomme à peine. Il développe, plutôt, une réflexion sur "l’idée de dictature" et explique qu'il ne traite pas de politique et pas davantage du Portugal: "Je ne saurais sans impertinence donner sur les actes de M. Salazar

\footnotetext{
4 José-Augusto França, "Culture, arts et lettres dans le Portugal des années quarante", in Lisbonne -Atelier du Lusitanisme Français, Paris, Presses Sorbonne Nouvelle, 2005, p. 22.

António Ferro, Salazar. Le Portugal et son chef (trad. de Fernanda de Castro, épouse d'António Ferro), précédé d'une "Note en guise de préambule sur 1'idée de dictature" de Paul Valéry, Paris, Flammarion, 1934. João Medina signale que Valéry se fit payer pour cette "préface". Il s'agissait, pour António Ferro, de saisir une occasion en or, d'associer, sur un plan international, l'écrivain français, académicien respecté, à Salazar, "mettant ainsi sur le même plan (ou au moins dans le même livre) le subtile auteur de L'âme et la danse et le provincial dictateur de Santa Comba" (João Medina, Salazar e a França, Lisbonne, Ática, 1977, p. 38).
} 
l'opinion de quelqu'un qui les ignore, puisque je n'ai pas été au Portugal”. Il refusera d'ailleurs, à plusieurs reprises, de s'y rendre. L'écrivain tente, avant tout, "de concevoir l'état naissant d'une dictature" et de comprendre comment naissent et meurent les régimes politiques. Pour Valéry, tout pouvoir est fiction qui n'existe et ne subsiste que par le mythe, qui n'existe qu'“ayant la parole pour signe”. Et la dictature elle-même "comporte de grandes illusions sur l'étendue et la profondeur de la puissance politique"6.

En 1941, Maurice Maeterlink écrira, à son tour, une "Introduction" à la version française du livre de Salazar, Une révolution dans la paix?.

La venue d'invités étrangers au Portugal de Salazar s'inscrit dans un projet bien plus ambitieux que José Rebelo analyse dans Formas de legitimação do poder no Salazarismo, où il explique que, si sur le plan interne, Salazar restreint ses contacts avec la communication sociale, évitant l'exposition et n'accordant des interviews ou des conférences de presse que lorsque cela s'impose ou sert son image et l'affirmation de son pouvoir, pour ce qui est de la construction de son image pour et à l'extérieur, la situation est tout autre: l'image de Salazar est entretenue, dans 1'opinion publique et dans le milieu intellectuel français, par de nombreuses références, presque toujours sous forme de louange, dans la presse et l'édition, surtout celles dominées par une droite maurrassienne et catholique. Une "certaine France", celle-là même qui verra, en Pétain, un père et un sauveur, se revoit aisément dans cette dictature paternaliste et traditionaliste, par opposition à d'autres dictatures plus démagogiques - celles d'un Hitler ou d'un Mussolini, figures grotesques et "plébéiens de trop mauvaise compagnie", selon le mot célèbre de Cicéron.

Situé à la pointe occidentale de l'Europe, le Portugal fut longtemps isolé, par deux obstacles, l'un géographique, l'autre mental. Entouré de deux côtés par l'immensité d'un océan et des deux autres par une Espagne qui surgit dans les esprits du reste de l'Europe, surtout des Français, comme infranchissable, il a souffert de l'amalgame avec l'identité culturelle espagnole. Daniel-Henri Pageaux observe que, pendant longtemps, la culture portugaise se dégage assez mal de l'emprise espagnole aux yeux des Français qui méconnaissent la langue

\footnotetext{
6 Paul Valéry, "Note en guise de préambule sur 1'idée de dictature", p. 10 (Cf. note 5).

7 António de Oliveira Salazar, Une révolution dans la paix, Paris, Flammarion, 1941 (Introd. de Maeterlink et trad. de Fernanda de Castro).
} 
portugaise et ne comprennent rien aux différences entre les deux langues et les deux cultures. Ignorance, mépris, indifférence, ce sont les trois vocables utilisés par Pageaux pour définir le regard français sur le Portugal: il rattache le mépris au $\mathrm{XVIII}^{\mathrm{e}} \mathrm{s}$. et l'ignorance et l'indifférence seraient le fait du $\mathrm{xIx}^{\mathrm{e}}$ et $\mathrm{xx}^{\mathrm{e}}$ siècles ${ }^{8}$.

C'est la Grande Guerre qui fait entrer le Portugal dans l'imaginaire français avec la mission militaire et diplomatique de Giraudoux à Lisbonne, en 1916. Son séjour de quatre mois lui inspirera un premier texte, "La Journée Portugaise", publié en 1920, dans Adorable Clio. Le Portugal devient, dès lors, objet d'un regard plus attentif de la part des Français. Jusqu'à l'arrivée de Salazar, son image à l'étranger était peu attrayante, oscillant entre une vision tragique d'un pays sans avenir, que l'on retrouve chez Antero de Quental, celle d'un pays de suicide, transmise par Miguel de Unamuno, et la vision satirique et amère de Eça de Queirós qui inspirait, en France, une ironie amusée et condescendante envers un peuple perçu comme indolent, insouciant, faisant des révolutions d'opérette" .

En 1930, Claude Farrère, qui est interviewé par António Ferro dans son livre Praça da Concórdia, publie Le Chef, un roman entièrement centré sur la figure de Salazar et qui ne laisse aucun doute sur les opinions politiques de l'auteur.

En 1935, le Portugal reçoit de nombreux d'écrivains d'expression française, invités par Ferro - François Mauriac, Jules Romains, Jacques Maritain, Georges Duhamel, Maeterlinck.

En 1938, Lacretelle et Robert de Traz font partie du jury du Grand Prix International Camões qui sera attribué à l'œuvre de Gonzague de Reynold - Le Portugal. Hans Jost, allemand "célèbre pour une pièce très violente contre la France", T.S. Eliot et Bontempelli figurent dans ce même jury, réuni pour couronner une œuvre étrangère à la gloire du Portugal salazariste. Zweig, au sommet de sa gloire, venait de publier Magellan et tout le désignait comme le vainqueur. Cependant, pour ne pas provoquer l'Allemagne, ce juif autrichien exilé fut tout simplement exclu. L'attention se porta alors sur Le nouveau Portugal de l'allemand F. Sieburg, connu pour son livre Dieu est-il français, et Portugal de Gonzague de Reynold. Sieburg se demandait si le Portugal de Salazar, pour demeurer fidèle à ses principes,

${ }^{8}$ Daniel-Henri Pageaux, Imagens de Portugal na cultura francesa, "Biblioteca Breve", ICLP, Lisbonne, 1983, p. 27-32.

9 Valery Larbaud, "Lettre de Lisbonne", Jaune Bleu Blanc, Paris, 'L'imaginaire', Gallimard, (1927), 1991, p. 924-925. 
pouvait continuer d'être francophile; Reynold défend les libertés nécessaires contre la liberté abstraite et impropre aux petits pays et va même jusqu'à faire l'éloge de l'analphabétisme et de la pauvreté qui est une vertu.

Dans Croisières en eaux troubles - "Quinze jours au Portugal" -, Jacques de Lacretelle donnera un témoignage fort intéressant d'un Portugal où il reviendra, en 1940, pour représenter officiellement la France aux célébrations du Double Centenaire, de même que Paul Hazard et Albert Kammerer. Charles Oulmont et Abel Bonnard seront là, eux aussi. Maeterlinck reviendra à cette même époque, bénéficiant des sympathies du Régime. Il sera reçu par Salazar, de même que le roumain Mircea Eliade qui, de 1941 à 1945, exerce les fonctions de Secrétaire de Presse à Lisbonne ${ }^{10}$.

En 1948, Pierre Benoît est invité par António Ferro alors qu'il est en route vers le Brésil. Il écrira même un roman étrange, Le Prêtre Jean, dédié au Secrétaire du SNI, dans lequel le roi D. Sebastião, disparu en 1578, au cour de la bataille d'Alcácer Quibir, revient sous la figure de Sidónio Pais.

En vérité, de nombreux intellectuels d'expression française, plus ou moins connus, visitent et/ou écrivent sur le Portugal de l'Estado Novo: ceux déjà cités mais aussi Charles Maurras, Jacques Bainville, Paul Descamps, Paul Sérant, Pierre Gaxotte, Léon de Poncins, Henri Massis, Jacques Maritain, Wladimir d'Ormesson, Gustave Thibon, J. Delebecque, Christian de Caters, Christine Garnier et tous proclament que le Portugal est «un exemple parfait de la modération, du bon sens et de la sagesse politique»"11 et que Salazar est un dictateur malgré lui, mais un dictateur-artiste, esthète et sculpteur, dont la matière-première, c'est l'homme ${ }^{12}$. Gonzague de Reynold l'affirme sans ambages dans Portugal, publié en 1936 et dont on retrouve, curieusement, des extraits dans un manuel scolaire de l'enseignement secondaire portugais, en 1940, pour l'apprentissage de la langue française:

Ce nouveau régime (celui de l'après 28 mai 1926) porte la marque personnelle et portera sans doute dans l'histoire le nom de Salazar. [...] L’homme nécessaire,

\footnotetext{
10 Cf. Mircea Eliade, Jornal Português. [1941-1945], Lisbonne, éd. Guerra e Paz, 2008.

11 José Rebelo, Formas de legitimação do poder no Salazarismo, Lisbonne, Livros e Leituras, 1998, p. 236.

12 Ibid, p. 232.
} 
certes, l'homme providentiel. [...] Je dirai que Salazar est le dictateur malgré lui, le dictateur par devoir. Il ne tient pas à la dictature, il est dénué de toute ambition personnelle, il porte le pouvoir comme un chrétien porte sa croix; on le sait, ce qui lui vaut un immense prestige moral. Il est en droit d'exiger beaucoup des autres lui qui exige tout de soi, et de tout demander, lui qui ne demande rien pour soi. [...] Salazar est donc un fils de la terre, presque un montagnard. Il tient de sa famille et de son milieu l'esprit pratique, l'esprit d'économie, la continuité dans l'effort, la prudence et la lenteur dans l'action, la réflexion calculée, la simplicité, même l'effacement, le manque de besoins, une certaine dureté jointe à la finesse et à un peu d'ironie, le sens de l'autorité, l'acharnement au travail, la volonté concentré, le respect des traditions, le patriotisme enraciné, la foi religieuse.13

Image d'un chef sobre et solitaire, à l'opposé même du caractère exalté de Mussolini et de Hitler, toujours représentés au milieu de foules bruyantes; vision on ne peut plus idéalisée de l'homme providentiel qui fait don de sa personne à son pays, se dévouant corps et âme à un peuple que lui seul peut protéger. Ce en quoi il préfigure Pétain!

On trouve, dans ce même manuel scolaire, un texte d'Abel Bonnard, maurrassien convaincu et ministre de l'Éducation nationale du gouvernement de Vichy (1942-1944) qui avoue, lors d'une visite au Portugal:

Au moment où je quitte le Portugal, je sens tous les liens qui m’attachent désormais à lui. Je l'aimais déjà avant d'y venir et mon séjour n’a fait que justifier et confirmer les sentiments qu'il m'avait inspiré de loin. J'aime cette terre ample et douce, ce peuple grave et bienveillant, cette élite qui accueille les Français en leur parlant leur langue avec une parfaite élégance. J'admire ce pays où l'ordre est noble, cette nation qui, riche d'un passé de gloire, entre d'un pas ferme dans son avenir.

La politique culturelle d'António Ferro réussira à imposer à l'Europe l'image d'un Portugal bucolique et heureux que la guerre de 1940 ne fera que renforcer. Ferro n'économise pas les moyens et multiplie les manifestations culturelles voyages, prix, fêtes, numéros spéciaux de revues, expositions. De toutes ces

13 Augusto César Pires de Lima, Carlos Santos, Recueil de Morceaux Choisis pour l'Enseignement Secondaire, II et III années, Porto, Domingos Barreira Editor, 1940. 
manifestations, on retiendra la Première Exposition Coloniale Portugaise à Porto (1934) qui souligne l'unité et la diversité d'un empire répandu "aux quatre coins du monde", mais surtout les somptueuses et gigantesques commémorations du Double Centenaire (1940) qui culminent avec l'Exposition du Monde Portugais, sur la Praça do Império. Inaugurée en 1940, elle attira un vaste nombre de visiteurs, nationaux et étrangers, consolidant, définitivement, la réputation d'António Ferro en tant qu'homme de culture. Le Portugal célébrait huit cents ans de nationalité (1140) et trois cents ans d'indépendance d'avec l'Espagne (1640). Mise en scène réconfortante du rêve mégalomane d'un dictateur-esthète, paternaliste et moraliste, souhaitant se rassurer et se prouver qu'il détenait un pouvoir sans limites, ces célébrations marquaient l'apogée du régime et visaient à renforcer le sentiment national de fierté patriotique. Le mythe du Cinquième Empire se trouvait, ainsi, pompeusement sacralisé sur l'autel des célébrations de 1940, alors que l'Europe était saccagée et déchirée par les forces maléfiques du nazisme. Indifférent à la souffrance d'une Europe au bord de l'abîme, le Portugal neutre de Salazar, île de bonheur et d'illusion, se complaît dans un semblant de paix et une insolite tranquillité.

\section{Simone de Beauvoir au Portugal: engagement et dissonance}

De tous les étrangers qui ont visité le Portugal jusqu'aux années 40, il en fut une qui vint déranger l'image idyllique, véhiculée jusqu'alors: Simone de Beauvoir, l'éternelle compagne de Sartre qui, par son exemple de vie et son engagement politique, devait s'affirmer comme l'égérie de la lutte pour la liberté et l'égalité des femmes au $x^{\mathrm{e}}$ siècle. Les images et les impressions qu'elle retiendra du Portugal de 1945 s'éloignent des stéréotypes transmis par Paul Morand, Jacques Chardonne, Valery Larbaud et bien d'autres qui ont exalté, à outrance, la douceur des mours, la beauté du paysage, le bleu du ciel et de la mer, la sensualité des varinas $^{14}$ de Lisbonne et de Ovar.

A la fin de la deuxième guerre mondiale, dans la France euphorique de la Libération, Simone de Beauvoir a 36 ans et se sent envahie par un énorme

14 Vendeuses de poisson. 
enthousiasme qu'elle décrira dans La Force des Choses I. Et si, jusqu'au début de la guerre, son existence était paisible, marquée par une enfance heureuse et bourgeoise, une jeunesse irrévérente, studieuse et brillante, marquée par la rencontre avec le jeune Sartre, le début d'une carrière d'enseignante et de nombreux voyages à l'étranger, la guerre éveillera en elle la nécessité de réfléchir sur la condition humaine et la responsabilité de l'intellectuel.

En février 1945, Simone de Beauvoir est invitée par l'Institut Français à Lisbonne, pour y proférer des conférences sur la vie intellectuelle en France durant l'Occupation. Profitant de cette visite, Albert Camus lui demande de rédiger quelques articles pour Combat, qu'il dirige. On s'étonne que le régime dictatorial de Salazar ait fait preuve d'une telle ouverture, s'agissant d'une personnalité déjà reconnue pour ses prises de position antifascistes et ses moeurs trop libérales et indépendantes pour les esprits portugais. Cependant, dans La force des choses I, on peut lire:

Pendant la guerre, le Portugal avait accordé toutes ses sympathies et certains appuis à l'Allemagne; Hitler vaincu, il se rapprochait de la France et c'est ainsi qu'il avait autorisé l'Institut français à patronner cette tournée. ${ }^{15}$

Il faut, avant tout, comprendre les rapports politiques qui s'étaient établis, pendant la guerre, entre le Portugal et la France, marqués par l'admiration réciproque de Salazar et Pétain, sans pour autant oublier que le Portugal «neutre» de Salazar avait été, pour tous ceux qui fuyaient les dangers du nazisme, une terre d'accueil. Simone de Beauvoir est donc reçue, en tant qu'élément distingué du Comité National des Ecrivains, dans le cadre d'un réajustement des relations franco-portugaises, souhaitable pendant cette période de l'immédiat après-guerre. Ayant établi des relations étroites avec le gouvernement de Vichy, Salazar éprouvait maintenant le besoin de travailler à une entente cordiale avec la France de De Gaulle.

Parallèlement aux raisons d'ordre politique, qui expliquent le séjour de Simone de Beauvoir au Portugal, il y en eut d'autres, d'ordre personnel: l'invitation lui fut adressée par son beau-frère, Lionel de Roulet, ancien élève de Jean-Paul Sartre:

15 Simone de Beauvoir, La force des choses I, Paris, Gallimard, 1963, p.46. 
Ma soeur avait épousé Lionel qui était maintenant attaché à l'Institut français de Lisbonne; il dirigeait une revue franco-portugaise, Affinidades. Il m’invita, au nom de l'Institut, à venir faire au Portugal des conférences sur l'occupation. Je me précipitai dans les bureaux des Relations culturelles et je demandai un ordre de mission. (pp. 33-34)

Arrivée à Lisbonne le 10 mai 1940 ${ }^{16}$, le jour même de l'invasion de la Hollande et de la Belgique par l'Allemagne et peu de jours avant l'invasion de Paris, Hélène finit par rester dans notre pays et s'y marie en 1942. Par la suite, elle aida son mari à fonder une revue qui devait exercer un rôle décisif dans la divulgation, au Portugal, d'auteurs français, déjà connus - ou sur le point de le devenir - et qui permit, en outre, à beaucoup d'écrivains portugais, de s'exprimer - Afinidades, Revista de Cultura Luso-Francesa. Dans Salazar em França, João Medina précise, dans une longue note:

La «revue de culture luso-française» Afinidades a été publiée à Lisbonne de 1942 à 1946, pendant vingt numéros. Son directeur était Francisco Fernandes Lopes, l'éditeur João Romualdo Mascarenhas et le chef de rédaction Lionel de Roulet (...) Elle a publié des textes de personnalités aussi diverses que Gide, Simone de Beauvoir, Ribeiro Couto, Moisés Amzalak, Abel Salazar, Paul Teyssier (l'excellent traducteur de Os Maias de Eça), Valéry, Adolfo Casais Monteiro, Colette, Mário Dionísio, Saint-Exupéry, Manuel da Fonseca, Malraux, Jaime Brasil, Camus, Joel Serrão, Eluard, Tomás Kim. (pp. 145-146)

A la liste proposée par João Medina nous ajouterons d'autres noms: des Portugais - Luís Forjaz Trigueiros, Fidelino de Figueiredo, Joly Braga Santos, João Gaspar Simões et Ramada Curto -, mais aussi des Français - Aragon, Giraudoux, Thierry Maulnier, Raymond Aron, Roger Caillois, François Mauriac, André Breton, Sartre, Simone de Beauvoir... celle-ci publie, dans le ${ }^{\circ} 12$ de Afinidades -"D'un nouvel humanisme" -, un article où elle expose ses réflexions existentialistes, prônant l'engagement politique de tous, et des écrivains en particulier, qui aura beaucoup inspiré nos intellectuels portugais et beaucoup inquiété Salazar et ses sbires:

16 Simone de Beauvoir, La force de l'âge II, Paris, Gallimard, 1963, pp. 560-561. 
[...] l'homme n'a pas besoin de paradis ni de repos. La suprême sagesse c'est pour lui de se donner à ses entreprises avec toute la passion, toute l'ardeur dont il est capable; et cependant de garder la certitude que, si important que soit le succès désiré, c'est la liberté même affirmée dans l'action qui est la valeur souveraine. Totalement engagé dans le monde, totalement libre au sein de cet engagement, tel doit être l'homme qui souhaite pleinement s'accomplir. Par son engagement il crée des fins, des buts, des motifs de haine, d'amour, d'action qui donnent un sens à sa vie; et s'il sait que sa liberté est le fondement suprême de ses fins, de ses actes, il n'a rien à craindre du monde ni même de la mort. ${ }^{17}$

La véhémence de ces opinions porte la marque de Dostö̈ewsky qui affirme que "chacun est responsable de tout, devant tous" et de Pascal pour qui «il est impossible de rester neutre». Elle critique l'égoïsme bourgeois de Flaubert, des naturalistes, de Mallarmé et de Proust et propose comme modèles d'un nouvel humanisme Saint-Exupéry, Malraux, Camus et Sartre. Une philosophie politique qui sera aussi celle des héros des Mandarins.

De ce premier voyage au Portugal en 1945 - elle y reviendra en 1975 -, nous trouvons l'écho dans plusieurs écrits qui concernent essentiellement trois genres: articles de journaux, écrits intimes et fiction - les articles sur le Portugal, publiés dans Combat; des extraits de ses Mémoires: La force de l'âge II; La force des choses I; des passages des Mandarins où elle "prête» à Henri ses souvenirs et ses images.

Le 27 février 1945, en fin de la journée, Simone prend le train qui la mènera de Paris à Madrid et à Lisbonne. Le 3 mars 1945, elle retrouve sa sour et son beau-frère et les premières heures furent dominées par l'euphorie des retrouvailles. Au faîte de l'enthousiasme provoqué par la nouveauté, Simone compare la capitale portugaise à Barcelone, Naples, Athènes et Marseille. Lisbonne était «une ville brûlante, fouettée par l'odeur de la mer; le passé soudain ressuscitait dans la nouveauté de ses collines et de ses promontoires, de ses tendres couleurs, de ses bateaux aux voiles blanches ${ }^{18}$. Elle se laisse d'abord séduire par le luxe et l'abondance des magasins, par la «débauche» qui la conduit à acheter sans

17 Simone de Beauvoir, "D'un nouvel humanisme", in Afinidades, n. ${ }^{\circ} 12$, Lisbonne, p. 12.

18 Simone de Beauvoir, La force des choses I, op cit, p. 44. 
mesure, un peu de tout: des sacs à main, des chaussures, des jupes, des robes, des chemisiers, des pulls et même un manteau de fourrure - et de nombreux cadeaux pour ses amis parisiens. Après six ans de rationnement et de privations de tout ordre, dans la France de l'Occupation, Lisbonne devait ressembler fort à la caverne d'Ali Baba.

Elle participe à un cocktail offert par l'Institut Français, "vêtue de frais», avec de nouveaux habits, assiste à des corridas, écoute le fado, se promène dans les jardins magnifiques de "Cintra", "parmi les camélias et les fougères arborescentes» et malgré les «jours sans voiture», elle a pu visiter l'Algarve (dans une voiture prêtée par l'Institut Français). La semaine suivante, elle prit le train pour Porto. Dans chaque gare, les mendiants envahissaient les wagons et à Porto, malgré les lumières de la nuit qui brillaient, et malgré la luminosité, belle et rouge du matin, entourée d'un brouillard blanc qui montait du Douro, Simone a vite découvert "la crasse humide des "îlots insalubres" grouillants d'enfants scrofuleux; des petites filles en haillons fouillaient avidement les poubelles ${ }^{19}$. Dans un élan de sincérité, l'écrivain avoue, cependant, que cette réalité sociale et économique derrière laquelle se cache un pouvoir politique dictatorial qui lui inspire une aversion profonde, ne l'a pas empêchée de "profiter» de tous les autres aspects que lui offrait le pays: elle buvait du vin vert et s'enivrait du bleu du ciel et de l'océan, des couleurs du lever et du coucher de soleil, elle s'émut devant les paysages d'un Minho fleuri, la beauté de Coimbra, Tomar, Batalha, Leiria et Óbidos. Le pittoresque, qu'elle voulait tant fuir, la rattrapa de nouveau à Braga où elle assista à des fêtes, des foires et des processions et où elle fut envahie, à nouveau, par une vague de consommation: elle acheta des foulards, des pots, des vases et des coqs en terre cuite et elle admira même les bœufs, magnifiques, avec des cornes en forme de lyre; le même pittoresque l'attendait à Nazaré, avec son port, ses bateaux et les couleurs des jupes des varinas. Mais, là encore, elle ne put s'empêcher de voir les mendiants, les gamins morveux, pieds-nus et les femmes qui portaient de lourds fardeaux sur le dos - à Nazaré aussi, le pittoresque s'est révélé impuissant à cacher la tristesse des regards. Malgré l'orgie de couleurs et de pompe religieuse, malgré l'harmonie apparente qui semblait

\footnotetext{
19 ibid., pp. 44-45.
} 
animer hommes et femmes, travaillant paisiblement dans les champs d'Alentejo et d'Algarve, elle avoue:

[...] je ne me laissais plus abuser; il y avait un mot dont je commençais à mesurer le poids: la faim. Sous les étoffes colorées, ces gens avaient faim; ils allaient pieds nus, le visage fermé, et dans les bourgades faussement pimpantes, je remarquai leurs regards hébétés; sous l'écrasant soleil, un désespoir sauvage les brûlait. ${ }^{20}$

Simone de Beauvoir raconte comment elle eut accès, par un médecin, à des informations sur la situation indigente de la santé et de l'hygiène, à des documents révélateurs des conditions de vie de la population - l'analphabétisme, les syndicats, la police, les prisons, la répression. On lui fit comprendre que le régime était bien assis, que rien ne menaçait le dictateur: le capitalisme anglais possédant de forts intérêts dans ce pays tel qu'il existait et les américains essayant de négocier, avec Salazar, la base militaire des Lages, aux Açores, seule l'opinion française pouvait, encore, être sensibilisée par la cruauté de la réalité portugaise.

Profondément choquée par tout ce qu'elle vit et sentit, et mue par le désir de respecter ses engagements envers tous les opposants au régime avec qui elle avait pu s'entretenir par l'intermédiaire de son beau-frère, dès son retour à Paris, elle entreprit la divulgation des faits et des aspects qu'elle voulait dénoncer. Et bien qu'ayant raconté son expérience portugaise dans plusieurs de ses livres de Mémoires et dans son roman, Les Mandarins, qui ne devait être publié que bien plus tard, elle l'a transposée, sur le champ, dans plusieurs articles, datés des 23 et 24 avril 1945, dans Combat, le célèbre journal fondé par la Résistance pendant l'Occupation. Souhaitant démystifier l'image d'un Portugal paradisiaque, après avoir vu de ses propres yeux la misère et l'injustice, Simone de Beauvoir publie plusieurs articles contre le régime de Salazar, lui faisant d'acerbes accusations. Afin de ne compromettre ni son beau-frère, ni sa sœur, ni ceux qu'elle avait côtoyés pendant son séjour, elle se choisit un pseudonyme - Daniel Secretan - pour signer les articles qui paraissent en première page du journal, avec de grands titres, expressément choisis pour attirer l'attention des lecteurs:

\footnotetext{
20 id., ibid.
} 
"Sur sept millions de portugais, il y en a 70.000 qui mangent"

"L'exploitation du peuple portugais par les privilégiés"

"Les riches portugais redoutent les portugais qui ont faim"

"Une Loi interdit de marcher pieds nus dans Lisbonne"

Leur contenu dérange le gouvernement portugais et menace de devenir un obstacle aux relations diplomatiques entre la France et le Portugal. L'ambassade du Portugal et les services de propagande portugais interviennent, exerçant des pressions sur la direction de Combat et Pascal Pia, qui avait remplacé Camus, absent en Afrique du Nord, en fit cesser la publication. Malgré ce cuisant échec, Simone de Beauvoir poursuivit sa mission, encore un temps, dans Volontés, revue dirigée par Collinet. Le ton subversif employé montre clairement son opposition à la ligne idéologique de Salazar et devait contribuer à une certaine démystification d'un régime qui, comme l'a démontré José Rebelo, a toujours joui de beaucoup d'adeptes véhéments, à l'étranger en général, et en France, en particulier. Simone commence, d'ailleurs, ainsi, l'un de ses articles:

On m’avait dit: "Certes le régime de Salazar est un régime autoritaire, mais c'est un autoritarisme tempéré, paternel, qui a su éviter les excès du fascisme, et l'œuvre qu'a accomplie Salazar le justifie d'avoir pris le pouvoir: ce qu'il a fait pour le Portugal est immense». J'ai visité le Portugal: j'ai cherché à voir ce que M. Salazar avait fait pour son peuple. Et j'ai vu. ${ }^{21}$

L’image qui en ressort est celle d'un Portugal misérable, opprimé, en rien semblable au tableau idyllique tracé par tant d'intellectuels français qui y étaient venus avant elle, et même après. Elle dresse un tableau accablant, des statistiques à l'appui, d'un pays dominé par une machine politique anti-démocratique et oppressante: faim, mendicité des enfants, prostitution, exploitation des plus indigents, insalubrité, emprisonnement et torture des opposants au régime, bafouant les droits essentiels de l'être humain. Pour ce qui est des Mandarins, le roman fut interdit par la censure, au Portugal, lors de sa parution, en 1953. Sa traduction et publication portugaises n'auront lieu qu'en 1975, peu de temps après

21 Simone de Beauvoir, "Le Portugal sous le régime de Salazar", in Francis, Claude et Gontier, Fernande, Les écrits de Simone de Beauvoir. La vie - L'écriture, Paris, N.R.F., Gallimard, 1979, p. 317. 
la chute de la dictature provoquée par la Révolution des oillets - au moment où, curieusement, Simone de Beauvoir revient au Portugal, en compagnie de Jean-Paul Sartre, cette fois-ci.

\section{Conclusion}

Le Portugal de Salazar semble répondre aux attentes des Français qui ressentent, dans leur majorité, une certaine crainte vis-à-vis des autres dictatures. "La république des instituteurs" qu'est la France de la IIIe République, regarde sans méfiance "la dictature douce d'un professeur" qui semble bien éloignée de la violence et des excès des autres régimes totalitaires, bolcheviques ou fascistes, véritables menaces pour l'ordre social et la paix mondiale. L'homme providentiel qui a remis de l'ordre dans un Portugal discrédité rappelle les deux figures centrales de l'imaginaire français: Pétain et De Gaulle. La séduction ressentie par les Français devant le mythe de Salazar s'explique, peut-être, par le fait que la France était, elle-même, un pays profondément terrien, méfiante vis-à-vis de la société industrielle et moderne. En même temps, la "fille aînée de l'Eglise" retrouve, dans l'Estado Novo, son propre héritage idéologique issu de l'Action Française, de Charles Maurras, "la seule idéologie française originale" selon Max Weber. Le Portugal est une sorte de mémoire historique d'un temps dont les Français ressentent la nostalgie et le dernier témoin d'un ancien monde, mythique, celui d'avant "le désenchantement du Monde" dont parle Max Weber.

Limage mythifiée d'une nation bucolique et heureuse va dominer la vision française du Portugal jusqu'à la Révolution des oeillets. Pendant l'Estado Novo, le regard des étrangers n'en donna qu'une image trop idéalisée, donc déformée, manipulée par des idéaux politiques et l'influence de "la politique de l'esprit" d'António Ferro. Certes, il y aura le témoignage dissonant de Simone de Beauvoir qui s'attardera sur la misère du peuple et les marques visibles d'un régime autoritaire, mais c'est encore cette vision, entachée de douceur et d'un certain exotisme, qui revient, d'abord chez des maurrassiens tels Chardonne et Michel Déon et plus tard, chez Mandiargues ou Pierre Kyria. Toujours et encore, une représentation idéalisée issue de Philippe Soupault, Valery Larbaud ou Paul Morand... 


\section{Mythification de Salazar en France - I}

\begin{tabular}{|c|c|}
\hline Nom & QEuvres \\
\hline Christine Garnier & - Vacances avec Salazar, Paris, Bernard Grasset, 1952. \\
\hline Charles Maurras & $\begin{array}{l}\text { Chef de file de l'Action Française, principal organe de diffusion de l'image } \\
\text { de Salazar et noyau dur du réseau salazariste en France. Maurras y a écrit } \\
\text { plusieurs articles sur Salazar et le Portugal. }\end{array}$ \\
\hline Jacques Bainville & - Les dictateurs, Paris, Editions Denoël et Steele, 1936. \\
\hline Henry Massis & $\begin{array}{l}\text { - Chefs, Paris, Plon, } 1939 . \\
\text { - Salazar face à face, trois dialogues politiques, Paris-Genève, La Platine, } \\
\text { 1961. } \\
\text { - Collaboration éparse dans la revue Itinéraires. }\end{array}$ \\
\hline Pierre Gaxotte & $\begin{array}{l}\text { - "Réflexions en marge de la révolution nationale portugaise" (précède } \\
\text { l'édition française de l'essai de Salazar Principes d'Action, Paris, Librairie } \\
\text { Arthème Fayard, 1956). } \\
\text { - Article dans le journal La Vie Française ("D.N.", 05/06/53). }\end{array}$ \\
\hline Gustave Thibon & $\begin{array}{l}\text { - "Le Président Salazar" (accompagne Principes d'Action et est partielle- } \\
\text { ment reproduit dans la revue L'Ordre Français, septembre-octobre 1970). }\end{array}$ \\
\hline Léon de Poncins & $\begin{array}{l}\text { - Le Portugal renâ̂t, Paris, Gabriel Beauchesne et ses fils, } 1936 . \\
\text { - "Décadence et Redressement du Portugal", in L'Ordre Français (septembre- } \\
\text {-octobre 1970). } \\
\text { - Série d'articles dans le journal Le Jour ("D.N.", 25, } 28 \text { et } 30 \text { juin et } 4 \text { juillet } \\
\text { 1935). }\end{array}$ \\
\hline J. Delebecque & $\begin{array}{l}\text { - Plusieurs articles dans Action Française (février 1935; mars et novembre } \\
\text { 1937; octobre 1942). }\end{array}$ \\
\hline Christian de Caters & $\begin{array}{l}\text { - Portraits du Portugal, Paris, Librairie Plon, } 1940 . \\
\text { - Article dans la revue Regards sur le Monde, mars } 1935 \text { ("D.N.", 22/03/35). } \\
\text { - "Un homme a rétabli le prestige de son pays", in Le Jour, novembre } 1936 \\
\text { ("D.N.", 05/12/36). } \\
\text { - Série d'articles dans La Revue Française de l'Elite: } \\
\text {-"Le Maréchal Carmona tel que je l'ai connu" (mai 1951) } \\
\text {-"Congrès au bord du Tage" (juin 1951) } \\
\text {-"La pensée du Président Salazar" (novembre 1962) } \\
\text { - "Styles d'hier", in Plaisir de France (juillet-août 1953) }\end{array}$ \\
\hline
\end{tabular}




\section{Mythification de Salazar en France - II}

\begin{tabular}{|c|c|c|}
\hline & Nom & Cuvres \\
\hline \multicolumn{2}{|c|}{ A. T'serstevens } & $\begin{array}{l}\text { - L'Itinéraire portugais, Paris, Grasset, } 1940 . \\
\text { - "Salazar rénovateur du Portugal", in Revue des deux mondes } \\
\text { (décembre 1939). }\end{array}$ \\
\hline \multicolumn{2}{|c|}{ Gonzague de Reynold } & $\begin{array}{l}\text { - Portugal, Paris, Editions SPES, 1936. (Grand Prix International } \\
\text { Camões!) } \\
\text { - Préface à Enquête à Goa de René Lombard. }\end{array}$ \\
\hline \multicolumn{2}{|c|}{ Maurice Martin du Gard } & $\begin{array}{l}\text { - Lettres Portugaises (reprises dans Un Français en Europe, } \\
\text { Paris, Flammarion, 1935). }\end{array}$ \\
\hline \multicolumn{2}{|c|}{ Charles Oulmont } & $\begin{array}{l}\text { Il écrit plusieurs articles sur Salazar et le Portugal, où il est } \\
\text { invité à plusieurs reprises par Ferro ( La Grande Revue, mars } \\
\text { 1936: "Regards sur les écrivains portugais"; Revue Hebdoma- } \\
\text { daire, avril 1936: "Le vrai Salazar". }\end{array}$ \\
\hline \multicolumn{2}{|c|}{ Abel Bonnard } & $\begin{array}{l}\text { Il représente l'Académie Française aux centenaires de l'Université } \\
\text { de Coimbra, remplaçant Paul Valéry (excusé). }\end{array}$ \\
\hline \multicolumn{2}{|c|}{ Pierre Benoit } & - Le Prêtre Jean, Paris, Albin Michel, 1952. \\
\hline \multicolumn{2}{|c|}{ Claude Farrère } & - Le chef, Paris, Flammarion, 1930. \\
\hline \multicolumn{2}{|c|}{ Marcel de Corte } & $\begin{array}{l}\text { - "L'œuvre et la personnalité de Salazar", in L'Ordre Français } \\
\text { (septembre-octobre 1970). }\end{array}$ \\
\hline \multicolumn{2}{|c|}{ Jacques Ploncard d'Assac } & $\begin{array}{l}\text { - Dictionnaire Politique de Salazar, Lisbonne, Edition du S.N.I. } \\
\text { en langue française, } 1964 . \\
\text { - Salazar, Paris, La Table Ronde, } 1969 . \\
\text { - "Salazar conscience de l'Occident", in Défense de l'Occident } \\
\text { (juin 1956). } \\
\text { - "La doctrine de Salazar", in L'Ordre Français (septembre- } \\
\text {-octobre 1970). } \\
\text { - Collaboration éparse dans la revue Ecrits de Paris. }\end{array}$ \\
\hline \multicolumn{2}{|c|}{ Louis Mégevand } & - Le vrai Salazar, Paris, Nouvelles Editions Latines, 1958. \\
\hline \multicolumn{2}{|c|}{ Paul Sérant } & - Salazar et son temps, Paris, Les Sept Couleurs, 1961. \\
\hline \multirow[t]{2}{*}{ Préfaciers } & Paul Valéry & $\begin{array}{l}\text { - "Au sujet de la dictature", in Regards sur le monde actuel et } \\
\text { autres essais, Paris, Gallimard, (1945), 2006. (préface à l'œuvre } \\
\text { d'António Ferro: Salazar. Le Portugal et son chef, trad. de Fer- } \\
\text { nanda de Castro, précédée d'une "Note sur 1'idée de dictature" } \\
\text { de Paul Valéry, Paris, Flammarion, 1934). }\end{array}$ \\
\hline & Maurice Maeterlink & $\begin{array}{l}\text { - Introduction à l'œuvre de Salazar, Une révolution dans la } \\
\text { paix, Paris, Flammarion, 1941. (trad. de Fernanda de Castro, } \\
\text { épouse d'António Ferro). }\end{array}$ \\
\hline
\end{tabular}




\begin{tabular}{|c|c|c|}
\hline Auteur & Titre & Source \\
\hline \multirow[t]{2}{*}{ Paul Bartel } & "Uma entrevista com o chefe do governo" & $\begin{array}{l}\text { in Figaro, Paris 15/08/33 } \\
\text { ("D.N.", 16/08/33) }\end{array}$ \\
\hline & "Oliveira Salazar dictateur mystique" & $\begin{array}{l}\text { in Le Mois, Paris, } \\
\text { septembre } 1933\end{array}$ \\
\hline Gerard Bauer & "A ditadura do silêncio" & $\begin{array}{l}\text { in L'Illustration, Paris } \\
\text { ("D.N.", 20/10/33) }\end{array}$ \\
\hline $\begin{array}{l}\text { Frédéric } \\
\text { Lefèvre }\end{array}$ & $\begin{array}{l}\text { "Une heure avec M. Oliveira Salazar - professeur } \\
\text { et dictateur mystique" }\end{array}$ & $\begin{array}{l}\text { in Les Nouvelles Littéraires } \\
\text { Paris } 1935\end{array}$ \\
\hline $\begin{array}{l}\text { Raymond } \\
\text { Recouly }\end{array}$ & "Le dictateur silencieux" & $\begin{array}{l}\text { in Gringoire, Paris } \\
26 / 02 / 37\end{array}$ \\
\hline Emile Schreiber & Le Portugal de Salazar & $\begin{array}{l}\text { Denoël, Paris, 1938, pp. } \\
\text { 53, 54-64 }\end{array}$ \\
\hline $\begin{array}{l}\text { André } \\
\text { Villeboeuf }\end{array}$ & Le coq d'Argent & $\begin{array}{l}\text { Les éditions de France, } \\
\text { Paris, 1939, pp. 112-115 }\end{array}$ \\
\hline \multirow[t]{2}{*}{$\begin{array}{c}\text { Charles } \\
\text { Chesnelong }\end{array}$} & Salazar & $\begin{array}{l}\text { Editions Baudinière, Paris, } \\
\text { 1939, pp. 211-214 }\end{array}$ \\
\hline & "Uma hora com Salazar" & $\begin{array}{l}\text { in Le Temps, Paris ("D.N.", } \\
07 / 08 / 42 \text { ) }\end{array}$ \\
\hline $\begin{array}{l}\text { Robert de } \\
\text { Saint-Jean }\end{array}$ & Carrefour & Paris ("D.N.", 27/03/52) \\
\hline Daniel Rops & France-Illustration & Paris ("D.N.", 08/06/52) \\
\hline Yves Delbars & "Caso humano" & $\begin{array}{l}\text { in Combat, Paris ("D.N.", } \\
26 / 05 / 53 \text { ) }\end{array}$ \\
\hline Coronel Rémy & "Jornal de uma viagem ao país de Salazar" & $\begin{array}{l}\text { in Aspects de la France, } \\
\text { Paris ("D.N.", 07/01/54) }\end{array}$ \\
\hline $\begin{array}{l}\text { V. Vernier } \\
\text { Contrepied }\end{array}$ & Charme et sagesse du Portugal & $\begin{array}{l}\text { Editions Eurafricaines, } \\
\text { Paris, 1956, pp. 106-113 }\end{array}$ \\
\hline
\end{tabular}




\section{BIBLIOGRAPHIE}

BEAUVOIR, Simone (de), (1954), Les Mandarins, Paris, Gallimard

, (1960), La force de l'âge I et II, Paris, Gallimard.

, (1963), La force des choses, Paris, Gallimard.

(1979), "Le Portugal sous le régime de Salazar", in Gontier, Fernande, Les écrits de Simone de Beauvoir. La vie - L'écriture. Appendice: Textes inédits ou retrouvés, Paris, Gallimard, 'N.R.F.', pp. 317-323.

ELIADE, Mircea, (2008), Jornal Português. [1941-1945], Lisbonne, éd. Guerra e Paz.

FERRO, António, (1934), Salazar. Le Portugal et son chef (trad. de Fernanda de Castro, précédé d'une "Note sur 1'idée de dictature" de Paul Valéry), Paris, Flammarion.

, (1933), Salazar, o homem e a sua obra, Lisbonne, Fernando Pereira Editor, 1982.

GARNIER, Christine, (1955), Vacances avec Salazar, Paris, Bernard Grasset, 1952 (édition originale, contenant des photos de Salazar avec Christine Garnier, prises par l'agent de la PIDE, Rosa Casaco); Férias com Salazar (traduction portugaise), Lisbonne, Parceria A. M. Pereira, (réédité en 2002, avec une préface de Fernando Rosas et des cartoons d'António).

LARBAUD, Valery, (1991), Jaune Bleu Blanc, Paris, Gallimard, (1927).

MEDINA, João, (1977), Salazar em França, Lisbonne, Ática.

(2000), Salazar, Hitler e Franco. Estudos sobre Salazar e a ditadura, Lisbonne, Livros Horizonte.

Ó, Jorge Ramos do, (1999), Os anos de Ferro - O dispositivo cultural durante a "Política do Espírito" 1933-1949, Lisbonne, Editorial Estampa.

PAGEAUX, Daniel-Henri, (1983), Imagens de Portugal na cultura francesa, "Biblioteca Breve", ICLP, Lisbonne.

PENJON, Jacqueline, (2005), Rivas, Pierre (éds.), Lisbonne - Atelier du Lusitanisme Français, Paris, Presses Sorbonne Nouvelle.

REBELO, José, (1998), Formas de Legitimação do Poder no Salazarismo, Lisbonne, Livros e Leituras.

RIVAS, Pierre, (1977), "Idéologies réactionnaires et séductions fascistes dans le futurisme portugais"; in Lista, Giovanni (org.), Marinetti et le Futurisme, Lausanne, LÂAe de 1'Homme, pp. 181-190 et 284-288.

, (1988), "Le Portugal et le Brésil au miroir français (1880-1940) - L'île et le jardin", in Portugal Brésil France Histoire et Culture, Paris, Fondation Calouste Gulbenkian, Centre Culturel Portugais, pp. 137-153.

, (2002), "Salazar en France", in Martins, Otília Pires (coord.), Portugal e o "Outro", uma relação assimétrica?, Aveiro, CLC, Universidade de Aveiro, pp. 155-161.

, (2005), Diálogos Interculturais, São Paulo, Editora Hucitec.

SALAZAR, (1941), António de Oliveira, Une révolution dans la paix (Introd. de Maeterlink et trad. de Fernanda de Castro). Paris, Flammarion.

TRIGUEIROS, Luis Forjaz, (1939), Capital do Espirito, Lisbonne, (s/ed.). 